\title{
Empfehlungen der Schweizerischen Medizinischen Interfakultätskommission (SMIFK) betreffend die Medizinalberufe und den Sanitätsdienst der Armee
}

\footnotetext{
* Die Schweizerische Medizinische Interfakultätskommission (SMIFK) ist eine Arbeitsgemeinschaft der zuständigen medizinischen Fakultäten und anderen beteiligten oder sich interessierenden Organisationen.
}

\section{Einleitung}

Die Medizinischen Fakultäten haben zusammen mit ihren Partnern in der SMIFK* (Eidgenössische Behörden, FMH, Naturwissenschaftliche Fakultäten, Praktizierende Ärzte, Studenten, u.a.) sowie Vertretern des Sanitätsdienstes und der Untergruppe Personelles der Armee ein Konzept zur Verbesserung der Ausbildung und des Bestandes der Militärärzte erarbeitet und schlagen, unter anderem, die Schaffung einer eidgenössischen interuniversitären Institution zur Ausbildung in Militär- und Katastrophenmedizin vor. Dadurch sollte die Schweiz in der Zukunft über eine grössere Zahl von in militär- und katastrophenmedizinischen Aspekten ausgebildeten Ärztinnen und Ärzten verfügen können. Gleichzeitig könnten diese Ärztinnen und Ärzte den dringend notwendigen Kern einer medizinischen Kriseninterventionskraft für besondere Hilfeleistungen im In- und Ausland bilden.

Synergistisch dazu interessiert sich auch das Schweizerische Korps für humanitäre Hilfe an einer Kooperation in der Nachdiplomausbildung seines medizinischen Fachpersonals in Katastrophenmedizin.

Der Aufbau der Schweizerischen Integrierten Akademie für Militär- und Katastrophenmedizin, in Zusammenarbeit mit dem Sanitätsdienst der Armee und den Medizinischen Fakultäten, wird von der FMH-Verbindung der Schweizer Ärztinnen und Ärzte, vom Bundesamt für Gesundheit und von den Partnern des Koordinierten Sanitätsdienstes unterstützt.

\section{Vorbemerkung}

Im nachfolgenden Text wird die männliche Form stellvertretend für die weibliche und männliche Bezeichnung verwendet.

\section{Empfehlungen}

\section{Sanitätsdienst und Armee XXI}

1. Frühzeitige Ermutigung der Kandidaten einer Ausbildung im Bereich des Gesundheitswesens, sich für eine kombinierte militärische und zivile Karriere zu interessieren.

2. Aufrechterhaltung eines Sanitätsdienstes von vertrauenswürdiger Qualität in der Armee XXI, entsprechend vergleichbaren Armeen anderer Länder, unter Einschluss der medizinischen und pharmazeutischen Dienste.

3. Übertragung der Verantwortung für den Sanitätsdienst soweit möglich auf allen Stufen an Personen mit professioneller Funktion im Gesundheitswesen. Bedarfsweise Finanzierung von vertraglich verpflichteten zivilen Ärzten oder Militärärzten, um den Mangel an Militärärzten, die obligatorische und freiwillige Dienstleistungen erbringen, zu entschärfen.

4. Aufbau einer Schweizerischen Integrierten Akademie für Militär- und Katastrophenmedizin.

5. Gleichbehandlung von Medizinern und übrigem Fachpersonal aus dem Gesundheitswesen mit anderen Berufsgattungen: Abschaffung der obligatorischen Pflicht zur militärischen Weiterausbildung.

6. Mediziner, die nicht bereit sind, sich einer militärischen Weiterausbildung zu unterziehen, sollen unabhängig vom Grad eingesetzt werden können, um den Sanitätsdienst der Armee punktuell verstärken oder ergänzen zu können. Das Gradsystem der Armee für das medizinische Personal muss revidiert werden, ebenso wie die Bedingungen, die erlauben, den Grad des Sanitätsoffiziers zu erreichen. 
7. Möglichkeit für die Mediziner, bis zu max. 75 Prozent ihrer Diensttage, die über dem Soll der Dienstleistungspflicht von Soldaten liegen, in zivilen Ausbildungsstätten oder anlässlich von Ausbildungseinsätzen zu leisten, die beide zivil und militärisch anerkannt sind.

8. Änderung und Anpassung des Dienstalters für Mediziner und Fachpersonal aus dem Gesundheitswesen, die einer langen Ausbildung und langjähriger praktischer Erfahrung bedürfen. Eine einheitliche Altersgrenze von 65 Jahren für Militärärzte, die bereit sind, freiwillig Dienst zu leisten.

9. Schaffung eines Modells für die Laufbahn der Militärärzte, das alle Diensttage verbunden mit dem Studium und der Weiterbildung einschliesst.

\section{Koordination und Harmonisierung der medizinischen und der militärischen Ausbildung}

1. Koordination des Beginns und der Dauer des akademischen Jahres der medizinischen Fakultäten.

2. Resultate der Abschlussprüfungen der Mittelschulen sollten spätestens 10 Wochen vor Beginn des akademischen Jahres vorliegen.
3. Ab dem dritten Studienjahr Festlegung eines einheitlichen studienfreien Zeitraums, mindestens alle 2 Jahre für mindestens 8 Wochen, um Militärdienst leisten zu können.

4. Vertikale (zeitliche) und horizontale (inhaltliche) Koordination der Ausbildungsprogramme der Fakultäten und des Sanitätsdienstes der Armee unter gegenseitiger Anerkennung.

5. Verstärkung der Funktion der Verbindungsperson «Armee» an den medizinischen Fakultäten als Kontakt- und Koordinationsstelle.

6. Obligatorische Ausbildung in Humanitärund Katastrophenmedizin an den Fakultäten, koordiniert mit der militärmedizinischen Ausbildung.

7. Institutionalisierung der Unterkommission SMIFK-Armee als permanentes Kontroll- und Koordinationsorgan der SMIFK zwischen Armee und Fakultäten. 